\title{
Gravitational couplings of intrinsic spin
}

This article has been downloaded from IOPscience. Please scroll down to see the full text article.

2000 Class. Quantum Grav. 172399

(http://iopscience.iop.org/0264-9381/17/12/312)

View the table of contents for this issue, or go to the journal homepage for more

Download details:

IP Address: 128.206.162.204

The article was downloaded on $27 / 09 / 2010$ at $14: 42$

Please note that terms and conditions apply. 


\title{
Gravitational couplings of intrinsic spin
}

\author{
Bahram Mashhoon \\ Department of Physics and Astronomy, University of Missouri-Columbia, Columbia, MO 65211, \\ USA
}

Received 27 March 2000

\begin{abstract}
The gravitational couplings of intrinsic spin are briefly reviewed. A consequence of the Dirac equation in the exterior gravitational field of a rotating mass is considered in detail, namely, the difference in the energy of a spin- $\frac{1}{2}$ particle polarized vertically up and down near the surface of a rotating body is $\hbar \Omega \sin \theta$. Here $\theta$ is the latitude and $\Omega=2 G J /\left(c^{2} R^{3}\right)$, where $J$ and $R$ are, respectively, the angular momentum and radius of the body. It seems that this relativistic quantum gravitational effect could be measurable in the foreseeable future.
\end{abstract}

PACS numbers: 0420C, 0365, 0480C

\section{Introduction}

About 40 years ago, Kobzarev and Okun [1] considered the theoretical possibility that a nuclear particle may possess a gravitoelectric dipole moment. This would lead to a violation of the equivalence principle through an interaction of the form $H_{\text {int }}=A \sigma \cdot g$, where $A$ is an amplitude, $\sigma$ is the particle spin and $\boldsymbol{g}$ is the gravitational acceleration due to a massive body such as the Earth. Similar spin-gravitoelectric couplings of the form $f(r) \sigma \cdot \hat{r}$ have been considered by a number of authors in connection with the possible breakdown of parity and time-reversal invariance in gravitation [2]. Leitner and Okubo used the hyperfine splitting of the ground state of hydrogen to set an upper limit on the strength of such an interaction [2]. Meanwhile, Dabbs et al [3] studied the freefall of neutrons polarized vertically up and down in the gravitational field of the Earth and found no splitting in the gravitational acceleration greater than a few per cent of $g$. However, a few years later observational evidence was reported for the gravitoelectric dipole moment of the proton [4]. This was soon shown to be spurious by the experiments of Vasil'ev [5] and Young [6]. In particular, Young [6] placed an upper limit of $0.3 \mathrm{~Hz}$ on the gravity shift of the proton Larmor frequency in 1969. Finally, a significant upper limit of $10^{-4} \mathrm{~Hz}$ was placed on a possible shift of the deuteron Larmor frequency due to the Earth's gravitational field by Wineland and Ramsey [7] in 1972.

In 1989, the observation of an anomalous difference in the weight of mechanical gyroscopes rotating vertically upward and downward was reported [8]. Again, the existence of such a rotor weight change was soon contradicted by subsequent experiments [9].

The observational search for the role of intrinsic spin in the gravitational interaction as well as the spacetime torsion has continued and many significant experiments have been performed [10-16]. These experiments have also explored finite-range axion-like interactions, which could be of the $\hat{\boldsymbol{r}} \cdot \boldsymbol{\sigma}$ ('monopole-dipole') form as well as a linear combination of $\boldsymbol{\sigma}_{A} \cdot \boldsymbol{\sigma}_{B}$ and $\hat{\boldsymbol{r}} \cdot \sigma_{A} \hat{\boldsymbol{r}} \cdot \sigma_{B}$ ('dipole-dipole') form, and have placed useful restrictions on the parameters of 
such interactions. Indeed, the past few decades have witnessed the emergence of extremely precise measurement techniques [17] that make it possible to detect frequency shifts of the order of $10^{-9} \mathrm{~Hz}$, an improvement of five orders of magnitude over what was possible three decades ago [7].

The aim of the present paper is to discuss the gravitomagnetic coupling of intrinsic spin due to the fact that according to the standard theory a spinning particle possesses a gravitomagnetic dipole moment. This moment couples to the gravitomagnetic field of a rotating mass (such as the Earth) in complete analogy with the $-\boldsymbol{\mu} \cdot \boldsymbol{B}$ interaction in electrodynamics. Instead of treating the Dirac equation in the exterior gravitational field of a rotating mass, a heuristic derivation of this general interaction is given in sections 2 and 3 on the basis of the gravitational Larmor theorem. For a spin- $\frac{1}{2}$ particle near the surface of the Earth, the effect involves a frequency shift of the order of $10^{-14} \mathrm{~Hz}$. Section 4 contains a brief discussion of the prospects for the measurement of this relativistic quantum gravitational effect.

\section{Inertia of intrinsic spin}

Imagine an observer in a laboratory on the Earth using Earth-based coordinate axes to describe the results of measurements. The particles involved in the experiments on the rotating Earth are waves propagating in inertial spacetime and it is natural to assume that they would keep their polarization aspects fixed in the underlying inertial frame. As measured by the observer, however, such intrinsic spin must 'precess' in a sense opposite to the sense of rotation of the Earth. The Hamiltonian associated with such motion would be of the form $H=-\boldsymbol{\sigma} \cdot \boldsymbol{\Omega}$, where $\boldsymbol{\Omega}$ is the frequency of rotation of the laboratory frame. The existence of such a Hamiltonian would show that intrinsic spin has rotational inertia. In quantum mechanics, mass and spin characterize the irreducible unitary representations of the inhomogeneous Lorentz group. The inertial properties of mass are well known in classical mechanics through various translational and rotational acceleration effects. In quantum mechanics, the inertial properties of mass have been investigated experimentally by a number of authors [18]. It is therefore interesting to consider the inertial properties of spin [19].

The coupling of intrinsic spin with rotation indicated above may be illustrated by a simple example. Imagine an observer rotating counterclockwise with uniform frequency $\Omega$ about the direction of propagation of a plane linearly polarized monochromatic electromagnetic wave of frequency $\omega \gg \Omega$. For instance, the observer could be in an Earth-based laboratory and $\Omega$ would then be the frequency of the proper rotation of the Earth. We neglect gravitational effects in this section and consider all phenomena in a global inertial frame in Minkowski spacetime. Let the observer move on a circle of radius $r$ with speed $c \beta=r \Omega$ in the $(x, y)$-plane of the inertial frame and let the electric field of the wave be given by the real part of

$$
\boldsymbol{E}=E_{0} \hat{\boldsymbol{x}} \mathrm{e}^{-\mathrm{i} \omega t+\mathrm{i} k z},
$$

where $E_{0}$ is a constant amplitude, $\boldsymbol{k}=k \hat{z}$ is the wavevector and $\omega=c k$. From the viewpoint of the rotating observer, the direction of linear polarization that is fixed in the inertial frame must drift in a clockwise sense about the direction of propagation, i.e.

$$
\boldsymbol{E}=E_{0}\left(\cos \Omega t \hat{\boldsymbol{x}}^{\prime}-\sin \Omega t \hat{\boldsymbol{y}}^{\prime}\right) \mathrm{e}^{-\mathrm{i} \omega t+\mathrm{i} k z},
$$

where $\hat{x}^{\prime}=\hat{x} \cos \Omega t+\hat{\boldsymbol{y}} \sin \Omega t, \hat{\boldsymbol{y}}^{\prime}=-\hat{\boldsymbol{x}} \sin \Omega t+\hat{\boldsymbol{y}} \cos \Omega t$ and $\hat{\boldsymbol{z}}^{\prime}=\hat{z}$ denote the Cartesian coordinate axes in the rotating frame of the observer. Specifically, the two coordinate systems are related by a simple rotation such that

$$
\hat{\boldsymbol{x}}+\mathrm{i} \hat{\boldsymbol{y}}=\mathrm{e}^{ \pm \mathrm{i} \Omega t}\left(\hat{\boldsymbol{x}}^{\prime} \pm \mathrm{i} \hat{\boldsymbol{y}}^{\prime}\right) .
$$


The linearly polarized wave (1) is a coherent superposition of a right circularly polarized (RCP) wave and a left circularly polarized (LCP) wave, i.e.

$$
\boldsymbol{E}=\frac{1}{2} E_{0}(\hat{\boldsymbol{x}}+\mathrm{i} \hat{\boldsymbol{y}}) \mathrm{e}^{-\mathrm{i} \omega t+\mathrm{i} k z}+\frac{1}{2} E_{0}(\hat{\boldsymbol{x}}-\mathrm{i} \hat{\boldsymbol{y}}) \mathrm{e}^{-\mathrm{i} \omega t+\mathrm{i} k z} .
$$

From the viewpoint of the rotating observer, these eigenstates of the radiation field remain invariant,

$$
\boldsymbol{E}=\frac{1}{2} E_{0}\left(\hat{\boldsymbol{x}}^{\prime}+\mathrm{i} \hat{\boldsymbol{y}}^{\prime}\right) \mathrm{e}^{-\mathrm{i}(\omega-\Omega) t+\mathrm{i} k z}+\frac{1}{2} E_{0}\left(\hat{\boldsymbol{x}}^{\prime}-\mathrm{i} \hat{\boldsymbol{y}}^{\prime}\right) \mathrm{e}^{-\mathrm{i}(\omega+\Omega) t+\mathrm{i} k z},
$$

except that the frequency of the RCP component is perceived to be $\omega-\Omega$, while that of the LCP wave is perceived to be $\omega+\Omega$ with respect to inertial time $t$. The proper time of the observer is, however, $\tau=t / \gamma$, where $\gamma=\left(1-\beta^{2}\right)^{-1 / 2}$. Thus we find that the proper frequencies measured by the observer are

$$
\omega^{\prime}=\gamma(\omega \mp \Omega) \text {. }
$$

Here the Lorentz factor accounts for time dilation, which is all that should happen according to the transverse Doppler effect. Instead, we have in (6) the additional 'angular Doppler terms' $\mp \Omega$ that have the following physical origin: in an RCP (LCP) wave, the electric and magnetic fields rotate in the positive (negative) sense about the direction of propagation with frequency $\omega$. Since the observer rotates in the positive sense with frequency $\Omega$, it perceives the effective frequency of the RCP (LCP) wave to be $\omega-\Omega(\omega+\Omega)$. In the JWKB limit, $\omega \rightarrow \infty$ and the 'angular Doppler terms' disappear since $\mp \Omega / \omega \rightarrow 0$. Our heuristic treatment ignores certain relativistic corrections that are not essential for the purposes of this discussion. Writing equation (6) in terms of the photon energy as $E^{\prime}=\gamma(E \mp \hbar \Omega)$, we see that the deviation from the simple transverse Doppler effect is due to the coupling of the spin of a circularly polarized photon to the rotation of the observer, since a RCP (LCP) photon carries an intrinsic spin of $\hbar(-\hbar)$ along its direction of propagation [20]. These elementary considerations already contain the basic aspects of the phenomenon of spin-rotation coupling, as can be seen from the following discussion based on the theory of relativity [21].

The special theory of relativity consists of two main elements: the principle of relativity (i.e. Lorentz invariance) and the hypothesis of locality. The latter specifies what an accelerated observer measures by establishing a connection between the accelerated observer and an inertial observer. Indeed, it requires that an accelerated observer be at each instant locally equivalent to a momentarily comoving inertial observer. This is a non-trivial axiom since there exist definite acceleration scales of time and length that are associated with an accelerated observer. In the case under consideration, for example, the acceleration length of the rotating observer is $\mathcal{L}=c / \Omega$ and the corresponding temporal scale is $\mathcal{L} / c=\Omega^{-1}$. Moreover, an elementary application of the hypothesis of locality would imply that $\omega^{\prime}=\gamma \omega$ by the transverse Doppler effect, since the connection between the instantaneous inertial frame of the accelerated observer and our global inertial frame simply results in the standard Doppler and aberration formulae with a time-dependent velocity $c \boldsymbol{\beta}(t)$. On the other hand, it should be clear that to measure wave characteristics such as the frequency, one must observe at least a few periods of the oscillations of the wave before a determination of the frequency becomes even possible. In this way, the curvature of the observer's worldline would have to be taken into consideration, and hence the standard Doppler and aberration formulae of relativity theory are valid only to the extent that the period of the wave $T=2 \pi / \omega$ is negligible compared to $\Omega^{-1}$, i.e. $\Omega T=2 \pi \Omega / \omega \rightarrow 0$. In view of these remarks, it is therefore natural to apply the locality axiom only to the electromagnetic field; then, the measured field could be Fourier analysed, which is a non-local operation, to obtain the frequency and wavevector content of the field in the accelerated frame. This is indeed the physical basis for the extension of relativistic 
wave equations to accelerated systems; in fact, this extended hypothesis of locality for wave phenomena is equivalent to the assumption of minimal coupling. Using this approach, one finds that for $\omega \gg \Omega$, the standard Doppler and aberration formulae should be modified to

$$
\begin{aligned}
\omega^{\prime} & =\gamma(\omega-c \boldsymbol{\beta} \cdot \boldsymbol{k})-\gamma \hat{\boldsymbol{H}} \cdot \boldsymbol{\Omega}, \\
\boldsymbol{k}^{\prime} & =\boldsymbol{k}+(\gamma-1)(\hat{\boldsymbol{\beta}} \cdot \boldsymbol{k}) \hat{\boldsymbol{\beta}}-\frac{1}{c} \gamma \omega \boldsymbol{\beta}+\frac{1}{c} \gamma(\hat{\boldsymbol{H}} \cdot \boldsymbol{\Omega}) \boldsymbol{\beta},
\end{aligned}
$$

where $\hat{\boldsymbol{H}}= \pm \boldsymbol{c} \boldsymbol{k} / \omega$ is the unit helicity vector. One can then consider optical interferometry in a rotating frame that would be based on the spin of the photon in contrast to the Sagnac effect that is connected to its orbital angular momentum [21].

The general expression for spin-rotation coupling can be written as

$$
E^{\prime}=\gamma(E-\hbar M \Omega),
$$

where $M$ is the total (orbital plus spin) 'magnetic' quantum number along the axis of rotation; that is, $M=0, \pm 1, \pm 2, \ldots$ for a scalar or a vector field, while $M \mp \frac{1}{2}=0, \pm 1, \pm 2, \ldots$ for a Dirac field. In the JWKB approximation, equation (9) can be written as $E^{\prime}=\gamma(E-\boldsymbol{\Omega} \cdot \boldsymbol{J})$, where $\boldsymbol{J}=\boldsymbol{L}+\boldsymbol{S}=\boldsymbol{r} \times \boldsymbol{P}+\boldsymbol{S}$. Thus $E^{\prime}=\gamma(E-\boldsymbol{v} \cdot \boldsymbol{P})-\gamma \boldsymbol{S} \cdot \boldsymbol{\Omega}$, so that in the absence of intrinsic spin we recover the classical expression for the energy of a particle as measured in the rotating frame with $\boldsymbol{v}=\boldsymbol{\Omega} \times \boldsymbol{r}$. The energy and momentum of a spinning particle as measured by an accelerated observer are then

$$
\begin{aligned}
& E^{\prime}=\gamma(E-\boldsymbol{v} \cdot \boldsymbol{P}-\boldsymbol{S} \cdot \boldsymbol{\Omega}), \\
& \boldsymbol{P}^{\prime}=\boldsymbol{P}+(\gamma-1)(\boldsymbol{P} \cdot \hat{\boldsymbol{\beta}}) \hat{\boldsymbol{\beta}}-\frac{1}{c} \gamma E \boldsymbol{\beta}+\frac{1}{c} \gamma(\boldsymbol{S} \cdot \boldsymbol{\Omega}) \boldsymbol{\beta},
\end{aligned}
$$

using the same JWKB approach as in the derivation of equations (7) and (8). It follows that

$$
E^{\prime 2}-c^{2} P^{\prime 2}=m^{2} c^{4}-2 E(\boldsymbol{S} \cdot \boldsymbol{\Omega})+(\boldsymbol{S} \cdot \boldsymbol{\Omega})^{2} .
$$

These results reduce to the equations appropriate for light once we set $E=\hbar \omega, \boldsymbol{P}=\hbar \boldsymbol{k}, \boldsymbol{S}=$ $\hbar \hat{\boldsymbol{H}}$ and $m=0$.

Experimental evidence for helicity-rotation coupling exists in the microwave and optical regimes via the phenomenon of frequency shift of polarized radiation [19]. Moreover, there is observational evidence for the coupling of spin- $\frac{1}{2}$ particles with the rotation of the Earth [19]. The analogous gravitational coupling of intrinsic spin is considered in the next section.

\section{Spin-gravitomagnetic coupling}

To extend the physics of spin-rotation coupling to the gravitational field, one must resort to Einstein's heuristic principle of equivalence. It is possible to interpret this principle in the post-Newtonian approximation via the gravitational Larmor theorem [22]. Newton's law of gravitation is formally analogous to Coulomb's law of electricity; therefore, one may describe Newtonian gravitational effects in terms of a gravitoelectric field. The classical tests of general relativity are all due to post-Newtonian gravitoelectric corrections. However, any consistent framework that brings Newtonian gravitation and Lorentz invariance together must of necessity contain a gravitomagnetic field that would be due to a mass current. A direct measurement of the gravitomagnetic field of the Earth via the precession of superconducting gyroscopes in a polar orbit about the Earth is one of the goals of the Stanford gyroscope experiment (GP-B) planned for 2001 . 
In the linear approximation of general relativity, where gravitational effects are treated as linear perturbations in a global inertial frame in Minkowski spacetime, one can express the gravitational field equations as Maxwell's equations for the gravitoelectric field $\boldsymbol{E}_{g}$ and the gravitomagnetic field $\boldsymbol{B}_{g}$ once $\mathrm{O}\left(c^{-4}\right)$ terms are neglected in the post-Newtonian metric perturbations [22]. Specifically, we let $g_{\mu \nu}=\eta_{\mu \nu}+h_{\mu \nu}$, where $\eta_{\mu \nu}$ is the Minkowski metric and for the linear perturbation $h_{\mu \nu}$ we define $\bar{h}_{\mu \nu}=h_{\mu \nu}-\frac{1}{2} \eta_{\mu \nu} h_{\alpha}^{\alpha}$. Then $\bar{h}^{00}=4 \phi_{g} / c^{2}$, $\bar{h}^{0 i}=2 A_{g}^{i} / c^{2}$ and $\bar{h}^{i j}=\mathrm{O}\left(c^{-4}\right)$. Here $\phi_{g}(t, \boldsymbol{x})$ is the gravitoelectric potential and $\boldsymbol{A}_{g}(t, \boldsymbol{x})$ is the gravitomagnetic vector potential. That is, of the ten effective gravitational potentials $\bar{h}_{\mu \nu}$ in general relativity, we neglect the six spatial potentials $\bar{h}_{i j}$ as these are of $\mathrm{O}\left(c^{-4}\right)$ for nonrelativistic (astronomical) sources and from the remaining four potentials one can construct a consistent theory of gravitoelectromagnetism (GEM) in this approximation scheme. Let us note that $\bar{h}^{0 \mu}=2 c^{-2}\left(2 \phi_{g}, A_{g}\right)$, so that the Lorentz gauge condition $\bar{h}^{\mu \nu}{ }_{, v}=0$ reduces in this case to

$$
\frac{2}{c} \frac{\partial \phi_{g}}{\partial t}+\nabla \cdot A_{g}=0
$$

Thus $A^{\mu}=\left(2 \phi_{g}, \boldsymbol{A}_{g}\right)$ is the effective GEM potential and the spacetime metric is given by

$$
\mathrm{d} s^{2}=-c^{2}\left(1-\frac{2 \phi_{g}}{c^{2}}\right) \mathrm{d} t^{2}-\frac{4}{c}\left(\boldsymbol{A}_{g} \cdot \mathrm{d} \boldsymbol{x}\right) \mathrm{d} t+\left(1+\frac{2 \phi_{g}}{c^{2}}\right) \delta_{i j} \mathrm{~d} x^{i} \mathrm{~d} x^{j} .
$$

The analogy with electrodynamics turns out to be exact, except for the fact that the ratio of the gravitomagnetic charge to the gravitoelectric charge is two, $q_{B} / q_{E}=2$; that is, linear gravity is a spin-2 field in contrast to the spin-1 character of the electromagnetic field that implies $q_{B} / q_{E}=1$ for the Maxwell theory.

In electrodynamics, Larmor established a theorem regarding the local equivalence of magnetism and rotation for all charged particles with the same charge-to-mass ratio $\mathrm{q} / \mathrm{m}$. In fact, the electromagnetic field can be locally replaced by an accelerated frame with translational acceleration $\boldsymbol{a}_{L}=-(q / m) \boldsymbol{E}$ and rotational (Larmor) frequency $\boldsymbol{\omega}_{L}=q \boldsymbol{B} /(2 m c)$. In electrodynamics, $q / m$ can be positive, zero or negative; however, the gravitational chargeto-mass ratio is universal due to the experimentally well tested equivalence of gravitational and inertial masses. This leads directly to Einstein's principle of equivalence and hence a geometric theory of gravitation. Einstein's heuristic principle of equivalence traditionally involves the local equivalence of the gravitoelectric field with the translational acceleration of the 'Einstein elevator' in Minkowski spacetime. The interpretation of Einstein's principle in terms of the gravitational Larmor theorem would then involve, in addition, the local equivalence of the gravitomagnetic field with the Larmor rotation of the elevator as well.

Let us consider the exterior field of an almost spherical rotating astronomical body (such as the Earth) with GEM potentials

$$
\phi_{g} \simeq \frac{G M}{r}, \quad \boldsymbol{A}_{g} \simeq \frac{G}{c} \frac{\boldsymbol{J} \times \boldsymbol{r}}{r^{3}},
$$

where $M$ is the mass and $J$ is the angular momentum of the source. These potentials can be obtained from the electromagnetic analogy by assuming that the source has positive gravitoelectric charge $Q_{E}=M$ and gravitomagnetic charge $Q_{B}=2 M$. The GEM fields are then

$$
\boldsymbol{E}_{g}=-\nabla \phi_{g}-\frac{1}{2 c} \frac{\partial}{\partial t} \boldsymbol{A}_{g}, \quad \boldsymbol{B}_{g}=\nabla \times \boldsymbol{A}_{g} .
$$

The motion of test particles in the gravitational field of a rotating mass can be obtained from the Lorentz force law if we assume that for a test particle of inertial mass $m$ the gravitational 
charges are negative, i.e. $q_{E}=-m$ and $q_{B}=-2 m$, in order to take due account of the dominant gravitational attraction between the test particle and the source. It turns out that an ideal test gyroscope at rest outside the rotating source undergoes gravitomagnetic precession

$$
\frac{\mathrm{d} S}{\mathrm{~d} t}=\Omega_{P} \times S
$$

with frequency

$$
\Omega_{P}=\frac{1}{c} B_{g}=\frac{G J}{c^{2} r^{3}}[3(\hat{\boldsymbol{r}} \cdot \hat{\boldsymbol{J}}) \hat{\boldsymbol{r}}-\hat{\boldsymbol{J}}] .
$$

Imagine now that we replace the gravitomagnetic field by a rotating frame in the neighbourhood of the gyroscope. As referred to observers at rest in the rotating frame, the motion of the gyroscope would be the same as before if the observers rotate with Larmor frequency $\boldsymbol{\omega}_{L}=-\boldsymbol{\Omega}_{P}$. This relation is consistent with the Larmor formula $\boldsymbol{\omega}_{L}=q \boldsymbol{B} /(2 m c)$ once we set $q_{B}=-2 m$ and $\boldsymbol{B}_{g}=c \boldsymbol{\Omega}_{P}$ as in equation (18). Thus a consistent and complete gravitoelectromagnetic formalism can be developed along these lines [22].

In particular, the spin-rotation coupling can be extended to gravitomagnetism via the Larmor theorem with $\omega_{L}=-\Omega_{P}$. That is, the interaction of intrinsic spin with the gravitomagnetic field is given by the Hamiltonian $H=\sigma \cdot \Omega_{P}$, since this interaction in the Larmor frame would be $H=-\boldsymbol{\sigma} \cdot \boldsymbol{\omega}_{L}$ as described in section 2. Moreover, the Heisenberg equations of motion for the spin-gravity interaction $H=\sigma \cdot \Omega_{P}$ are formally the same as equations (17) and (18) for the precession of an ideal test gyroscope.

In classical electrodynamics, the magnetic dipole moment for a particle of mass $m$ and charge $q$ is given by $\boldsymbol{\mu}=q \boldsymbol{S} /(2 m c)$, where $\boldsymbol{S}$ is its orbital angular momentum. The energy associated with the interaction of this magnetic moment with a magnetic field $\boldsymbol{B}$ is $-\boldsymbol{\mu} \cdot \boldsymbol{B}$. Extending these notions to GEM with $q_{B}=-2 m$, we find that a gravitomagnetic dipole moment for a gyroscope of spin $S$ is $\boldsymbol{\mu}_{g}=-S / c$ and the energy of interaction with a gravitomagnetic field is $-\boldsymbol{\mu}_{g} \cdot \boldsymbol{B}_{g}=\boldsymbol{S} \cdot \boldsymbol{\Omega}_{P}$. A further extension of this result to the intrinsic spin of particles naturally leads to the interaction Hamiltonian $H=\sigma \cdot \Omega_{P}$. The gravitoelectric analogue of this interaction has already been discussed in section 1 ; that is, $H_{\mathrm{int}}=-\boldsymbol{d}_{g} \cdot \boldsymbol{E}_{g}$, where $d_{g}=A \sigma$ would be the hypothetical gravitoelectric dipole moment of a particle [1,2] and $\boldsymbol{E}_{g}=-\boldsymbol{g}$ from (15) and (16).

Let us imagine an experiment in a laboratory near the surface of an astronomical body (such as the Earth) involving the difference in the energy of a particle of spin $\sigma=s \hbar$ polarized vertically up and down (i.e. perpendicular to the surface). According to the spingravitomagnetic coupling, the result is

$$
E_{+}-E_{-}=2 s \hbar \Omega \sin \theta \text {. }
$$

Here $\theta$ is the geographic latitude (i.e. $E_{+}=E_{-}$at the equator) and $\Omega$ is the effective frequency associated with the gravitomagnetic field

$$
\Omega=\frac{2 G J}{c^{2} R^{3}},
$$

where $R$ is the mean radius of the body. Equation (19) expresses a relativistic quantum gravitational effect; indeed, one can write $\hbar \Omega=\left(2 c J / R^{3}\right) L_{P}^{2}$, where $L_{P}=\left(\hbar G / c^{3}\right)^{1 / 2}$ is the Planck length. Let us note that for the Earth $\hbar \Omega_{E} \simeq 2 \times 10^{-29} \mathrm{eV}$, while near the surface of Jupiter $\hbar \Omega_{J} \simeq 10^{-27} \mathrm{eV}$; similarly, for the Sun $\hbar \Omega_{S} \sim 10^{-27} \mathrm{eV}$, but for a neutron star $\hbar \Omega_{N S} \sim 10^{-14} \mathrm{eV}$.

It is important to point out that the spin-rotation-gravity coupling has appeared in the work of many authors who have studied wave equations in accelerated systems and gravitational 
fields [23, 24]. In particular, the $\sigma \cdot \Omega_{P}$ interaction under scrutiny in this work first appeared in the work of de Oliveira and Tiomno [23]. The observation of wave phenomena associated with such couplings was first investigated independently in connection with possible limitations of the general theory of relativity in [25]. Dynamics in electromagnetic fields can be generated by the transformation of the momentum via $p_{\mu} \rightarrow p_{\mu}-(q / c) A_{\mu}$, where $A_{\mu}=(-\phi, \boldsymbol{A})$ is the electromagnetic (EM) potential. The same holds in the GEM case, except that the analogue of $A_{\mu}$ is $\left(-2 \phi_{g}, \boldsymbol{A}_{g}\right)$. Let us consider, for instance, the motion of electromagnetic waves in the exterior field of a rotating mass. The effective gravitational charge in this case should be determined based on the fact that a photon of energy $\hbar \omega$ in 'cyclotron' motion has an effective inertial mass of $\hbar \omega / c^{2}$ and hence the effective GEM charges are $q_{E}=-\hbar \omega / c^{2}$ and $q_{B}=-2 \hbar \omega / c^{2}$. The eigenvalue problem in gravitomagnetic fields leads to discreteness properties for the modes reminiscent of the Fock-Darwin-Landau levels in a magnetic field. Imagine, for instance, the motion of electromagnetic waves in a gravitomagnetic field characterized by the magnitude of the effective 'cyclotron' frequency $\Omega_{c} \simeq 2 G J /\left(c^{2} r^{3}\right)$. It follows from the explicit solution of Maxwell's equations in this background [25] that the wavefunctions are proportional to Hermite polynomials. These polynomials vary over a harmonic characteristic length scale $l_{g}$ that is given by

$$
l_{g}=\frac{c}{\left(\omega \Omega_{c}\right)^{1 / 2}}
$$

for an electromagnetic mode of frequency $\omega$. If in this equation we set $\hbar \omega=m c^{2}$ and $\Omega_{c}$ as the cyclotron frequency in a magnetic field, we recover the magnetic length that is well known in the discussion of the motion of a charged particle of mass $m$ in a magnetic field. It is interesting to note that the gravitomagnetic acceleration length is given by $\mathcal{L}_{g}=c / \Omega_{c}$, so that the gravitomagnetic length (21) is the geometric mean of the reduced wavelength of radiation $\lambda$ and $\mathcal{L}_{g}$. The gravitomagnetic length $l_{g}$ is essentially the same as the radius of the 'cyclotron' orbit for a mode with frequency equal to the 'cyclotron' frequency $\left(\omega=\Omega_{c}\right)$. The eigenvalue spectrum clearly shows the existence of a gravitomagnetic coupling between the photon spin and the rotation of the source [25]. One can show that in the eikonal approximation the gravitational helicity-rotation coupling leads to a differential deflection of polarized radiation, thus violating the universality of freefall in a gravitational field beyond the geometric optics limit $[22,25]$. That the spin-gravity interaction violates the universality of freefall is already apparent from $H=\sigma \cdot \Omega_{P}$, since this Hamiltonian depends only on the spin of the particle and is independent of its mass.

Imagine, for instance, the scattering of electromagnetic radiation by a black hole (i.e. pure geometry free of matter). For a Schwarzschild black hole, the scattering amplitude is independent of the polarization of the incident radiation, hence the polarization properties of the radiation are preserved in the scattering process. For a Kerr black hole, however, the scattering amplitude is dependent upon the polarization of the incident radiation. It is possible to give only rough and partial estimates for the motion of wavepackets in a gravitomagnetic field $[25,26]$. The influence of helicity-rotation coupling on the gravitational deflection of electromagnetic radiation is rather weak and far below the existing observational upper limits [27], but could become important in future microlensing experiments with polarized radiation. To provide useful astrophysical estimates of the resulting polarization-dependent deflection of radiation, an eikonal approach has been developed for the motion of rays based on equations (7) and (8), i.e. $\omega(\boldsymbol{r}, \boldsymbol{k})=c k \pm \hat{\boldsymbol{k}} \cdot \Omega_{P}(\boldsymbol{r})$, so that the Einstein deflection is ignored for the sake of simplicity and only the helicity-rotation coupling is taken into account [22]. In this treatment, the total differential deflection of positive and negative helicity rays approaching the source together from asymptotic infinity and travelling to infinity after deflection vanishes in contrast 
to what is expected from the wave treatment; however, it is possible to obtain useful estimates for radiation originating near the source. For instance, consider radiation originating over a pole and propagating normal to the rotation axis with an impact parameter $D$; then, RCP and LCP waves separate by a total angle of $\delta \approx 4 \lambda G J /\left(c^{3} D^{3}\right)$ about the average Einstein deflection angle. A qualitative description of this effect is given in [28]. The gravitomagnetic splitting $\delta$ is small; it amounts to about 1 marcsec for $\mathrm{GHz}$ radio waves passing over a pole of a neutron star. In addition to this splitting, one expects a wavelength-independent gravitomagnetic rotation of the plane of polarization along a ray, i.e. the Skrotskii effect that is the gravitational analogue of the Faraday effect $[25,29]$. Moreover, the difference in the arrival times of positive and negative helicity radiation originating near a rotating mass and propagating freely outward to a distant point is estimated to be $T_{+}-T_{-}=-2 \lambda G \boldsymbol{J} \cdot r /\left(c^{4} r^{3}\right)$, where $r$ is the position vector of the point of origin of the radiation relative to the centre of the rotating source. This differential time delay due to the different phase speeds of RCP and LCP waves is too small to be measurable at present [22].

The violation of the universality of freefall is a wave effect, so that it vanishes in the $\lambda / \mathcal{L}_{g} \rightarrow 0$ limit. Consider, for instance, a spinning particle in a gravitomagnetic field with the interaction Hamiltonian $H=\sigma \cdot \Omega_{P}$. This potential energy is position dependent; therefore, there exists a gravitomagnetic Stern-Gerlach force $\boldsymbol{F}=-\nabla H$ acting on the particle that is independent of mass and hence violates the universality of the gravitational acceleration. Specifically,

$$
\boldsymbol{F}=\frac{3 G J}{c^{2} r^{4}}\{[5(\boldsymbol{\sigma} \cdot \hat{\boldsymbol{r}})(\hat{\boldsymbol{J}} \cdot \hat{\boldsymbol{r}})-\boldsymbol{\sigma} \cdot \hat{\boldsymbol{J}}] \hat{\boldsymbol{r}}-(\boldsymbol{\sigma} \cdot \hat{\boldsymbol{r}}) \hat{\boldsymbol{J}}-(\hat{\boldsymbol{J}} \cdot \hat{\boldsymbol{r}}) \boldsymbol{\sigma}\}
$$

so that the weight operator for the particle $W=m g-\boldsymbol{F} \cdot \hat{\boldsymbol{r}}$ is given by $W=m g-3 H / r$. If the spin is polarized vertically up or down in a laboratory near the Earth,

$$
W_{ \pm}=m g \mp \frac{3 s}{R} \hbar \Omega \sin \theta
$$

so that $W_{ \pm}=m g(1 \mp \epsilon)$, where $\epsilon$ can be expressed as

$$
\epsilon=6 s\left(\frac{I}{M R^{2}}\right)\left(\frac{\hbar \omega}{m c^{2}}\right) \sin \theta
$$

Here $J=I \omega, I$ is the moment of inertia and $\omega$ is the proper rotation frequency of the Earth. For a neutron near the Earth's surface, $\hbar \omega /\left(m_{n} c^{2}\right) \simeq 5 \times 10^{-29}$; hence, $\epsilon$ is too small to be measurable in the foreseeable future. It follows that for polarized materials the relevant $\epsilon$ is expected to be even smaller. Let us note that $\epsilon$ is directly proportional to $\hbar \omega /\left(m c^{2}\right)$, which can be expressed as the ratio of the Compton wavelength of the particle $(\hbar / m c)$ to the rotational acceleration length of the observer $(c / \omega)$. Indeed, the extended nature of the particle makes it possible for its intrinsic spin to couple to the spacetime curvature, resulting in a force $\boldsymbol{F}$ that has an exact analogue in the classical Mathisson-Papapetrou spin-curvature force [22, 28].

Thus far we have discussed the gravitomagnetic spin-rotation coupling in terms of a single rotating source such as the Earth. However, the universality of the gravitational interaction implies that the whole mass-energy content of the universe is involved in every physical experiment via the gravitational interaction. In classical physics, the gravitational force of the rest of the universe enters only through its gradients, which turn out to be rather small for experiments in the solar system. The situation is in general different in quantum physics, however. For instance, in the calculation of the spin-gravity coupling, the gravitomagnetic field generated by the total mass-energy current must be taken into account. This is a 
difficult problem; however, to obtain some idea of what is involved here we may use the linear approximation to write the interaction Hamiltonian as

$$
H=\frac{G}{c^{2}} \sum_{a} \frac{3\left(\boldsymbol{r}_{a} \cdot \boldsymbol{J}_{a}\right)\left(\boldsymbol{r}_{a} \cdot \boldsymbol{\sigma}\right)-r_{a}^{2}\left(\boldsymbol{J}_{a} \cdot \boldsymbol{\sigma}\right)}{r_{a}^{5}},
$$

where the sum is over all astronomical sources and $\boldsymbol{r}_{a}=\boldsymbol{x}_{a}-\boldsymbol{x}$ is the vector of relative separation between the particle of spin $\boldsymbol{\sigma}$ at $\boldsymbol{x}$ and the centre of mass of the source $a$. Equation (25) can be expressed as $H=c\left(\boldsymbol{\sigma} \cdot \partial_{\boldsymbol{x}}\right) \Phi_{g}$, where

$$
\Phi_{g}=\frac{G}{c^{3}} \sum_{a} \frac{\boldsymbol{J}_{a} \cdot \boldsymbol{r}_{a}}{r_{a}^{3}}
$$

is the net dimensionless scalar gravitomagnetic potential defined by $\boldsymbol{B}_{g}=c^{2} \nabla \Phi_{g}$. For a laboratory experiment near the Earth, it is simple to show that the net contribution due to the Sun, the Moon and the other planets is negligible. Therefore, to compute $\Phi_{g}$ one must investigate the cosmic mass-current distribution. This is a difficult observational problem and much remains unknown regarding the distribution of angular momentum in the universe. It is likely that over the largest scales no preferred sense of rotation would be discernible. These considerations lead one to surmise that near the Earth (or Jupiter) the main contribution to the Hamiltonian is simply due to the Earth (or Jupiter), though a completely satisfactory resolution is not available. Conversely, observational data regarding the gravitomagnetic spin-rotation coupling could in principle set limits on the cosmic mass-current distribution.

\section{Discussion}

The gravitational coupling of intrinsic spin with rotation has been described in this work and the consequences of the gravitomagnetic interaction $H=\sigma \cdot \Omega_{P}$ have been pointed out. In particular, the gravitomagnetic shift in the Larmor frequency of a nuclear particle has been estimated. Efforts are underway to improve the sensitivity of the measurement of such frequency shifts by several orders of magnitude. This could potentially make the effect measurable near the surface of Jupiter [30]. Let us recall that for Jupiter $\hbar \Omega_{J} \simeq 10^{-27} \mathrm{eV}$, corresponding to a gravitomagnetic Larmor shift of about $3 \times 10^{-13} \mathrm{~Hz}$. In view of the current interest in planetary exploration, it appears that the gravitomagnetic coupling of intrinsic spin with rotation could be measurable in the foreseeable future.

\section{Acknowledgments}

I am grateful to Friedrich Hehl and Michael Romalis for helpful discussions and correspondence.

\section{References}

[1] Kobzarev I Yu and Okun L B 1963 Sov. Phys.-JETP 161343

[2] Leitner J and Okubo S 1964 Phys. Rev. B 1361542

Hari Dass N D 1976 Phys. Rev. Lett. 36393

Peres A 1978 Phys. Rev. D 182739

Morgan T A and Peres A 1962 Phys. Rev. Lett. 979

[3] Dabbs J W T, Harvey J A, Paya D and Horstmann H 1965 Phys. Rev. B 139756

[4] Velyukhov G E 1968 JETP Lett. 8229

[5] Vasil'ev B V 1969 JETP Lett. 9175 
[6] Young B A 1969 Phys. Rev. Lett. 221445

[7] Wineland D J and Ramsey N F 1972 Phys. Rev. A 5821

[8] Hayasaka H and Takeuchi S 1989 Phys. Rev. Lett. 632701

[9] Faller J E, Hollander W J, Nelson P G and McHugh M P 1990 Phys. Rev. Lett. 64825

Quinn T J and Picard A 1990 Nature 343732

Nitschke J M and Wilmarth P A 1990 Phys. Rev. Lett. 642115

[10] Adelberger E G, Heckel B R, Stubbs C W and Rogers W F 1991 Ann. Rev. Nucl. Part. Sci. 41269

[11] Wineland D J et al 1991 Phys. Rev. Lett. 671735

[12] Venema B J et al 1992 Phys. Rev. Lett. 68135

[13] Ritter R C, Winkler L I and Gillies G T 1993 Phys. Rev. Lett. 70701

[14] Berglund C J et al 1995 Phys. Rev. Lett. 751879 Youdin A N et al 1996 Phys. Rev. Lett. 772170

[15] Ni W-T et al 1999 Phys. Rev. Lett. 822439 Chui T C P and Ni W-T 1993 Phys. Rev. Lett. 713247

[16] Vorobyov P V and Gitarts Ya I 1988 Phys. Lett. B 208146 Bobrakov V F et al 1991 JETP Lett. 53294 Vorob'ev P V 1994 JETP Lett. 59510

[17] Jacobs J P et al 1995 Phys. Rev. A 523521

[18] Werner S A, Staudenmann J-L and Colella R 1979 Phys. Rev. Lett. 421103 Rauch H and Werner S A 2000 Neutron Interferometry (Oxford: Clarendon) Atwood D K, Horne M A, Shull C G and Arthur J 1984 Phys. Rev. Lett. 521673 Bonse U and Wroblewski T 1983 Phys. Rev. Lett. 511401 Hasselbach F and Nicklaus M 1993 Phys. Rev. A 48143 Moorhead G F and Opat G I 1996 Class. Quantum Grav. 133129 Schwab K, Bruckner N and Packard R E 1997 Nature 386585 Gustavson T L, Bouyer P and Kasevich M A 1997 Phys. Rev. Lett. 782046

[19] Mashhoon B 1995 Phys. Lett. A 1989 Mashhoon B, Neutze R, Hannam M and Stedman G E 1998 Phys. Lett. A 24916

[20] Beth R A 1936 Phys. Rev. 50115

[21] Mashhoon B 1988 Phys. Rev. Lett. 612639 Mashhoon B 1992 Phys. Rev. Lett. 683812 Mashhoon B 1989 Phys. Lett. A 139103 Mashhoon B 1990 Phys. Lett. A 143176 Mashhoon B 1990 Phys. Lett. A 145147 Mashhoon B 1993 Phys. Rev. A 474498

[22] Mashhoon B 1993 Phys. Lett. A 173347 Mashhoon B, Gronwald F and Theiss D S 1999 Ann. Phys., Lpz. 8135 Mashhoon B, Gronwald F and Lichtenegger H I M 2000 Testing Relativistic Gravity in Space ed C Lämmerzahl, C W F Everitt and F W Hehl (Berlin: Springer) (Mashhoon B, Gronwald F and Lichtenegger H I M 1999 Preprint gr-qc/9912027)

[23] de Oliveira C G and Tiomno J 1962 Nuovo Cimento 24672 Mitskievich N V 1969 Physical Fields in General Relativity Theory (Moscow: Nauka) (in Russian) Schmutzer E 1973 Ann. Phys., Lpz. 2975

Barker B M and O'Connell R F 1975 Phys. Rev. D 12329 Schmutzer E and Plebański J 1977 Fortschr. Phys. 2537

[24] Hehl F W and Ni W-T 1990 Phys. Rev. D 422045 Cai Y Q and Papini G 1991 Phys. Rev. Lett. 661259 Cai Y Q and Papini G 1992 Phys. Rev. Lett. 683811 Anandan J 1992 Phys. Rev. Lett. 683809 Andretsch J and Lämmerzahl C 1992 Appl. Phys. B 54351 Silverman M P 1992 Nuovo Cimento D 14857 Huang J 1994 Ann. Phys., Lpz. 353 Soares I D and Tiomno J 1996 Phys. Rev. D 542808 Ryder L H 1999 Gen. Rel. Grav. 31775

[25] Mashhoon B 1974 Nature 250316 Mashhoon B 1974 Phys. Rev. D 101059 Mashhoon B 1975 Phys. Rev. D 112679

[26] Damour T and Ruffini R 1974 C.R. Acad. Sci. A 279971 
de Logi W K and Kovacs S J Jr 1977 Phys. Rev. D 16237

Leahy D A 1982 Int. J. Theor. Phys. 21703

Mashhoon B 1987 Phys. Lett. A 122299

Futterman J A H, Handler F A and Matzner R A 1988 Scattering from Black Holes (Cambridge: Cambridge University Press)

Feng L L and Lu T 1991 Class. Quantum Grav. 8851

Carini P, Feng L L, Li M and Ruffini R 1992 Phys. Rev. D 465407

[27] Harwit M et al 1974 Nature 249230

Dennison B, Dickey J and Jauncey D 1976 Nature 263666

Dennison B et al 1978 Nature 27333

[28] Mashhoon B 1999 Gen. Rel. Grav. 31681

[29] Kopeikin S and Mashhoon B 2000 Preprint

[30] Romalis M 2000 Private communication 